\title{
Distal-less homeobox 5 is a master regulator of the osteogenesis of human mesenchymal stem cells
}

\author{
JUNE SEOK HEO ${ }^{1,2}$, SEUNG GWAN LEE ${ }^{3}$ and HYUN OK KIM ${ }^{2,4}$ \\ ${ }^{1}$ Department of Integrated Biomedical and Life Sciences, College of Health Science, Korea University, Seoul 02841; \\ ${ }^{2}$ Cell Therapy Center, Severance Hospital, Seoul 03722; ${ }^{3}$ Department of Health and Environmental Science, \\ College of Health Science, Korea University, Seoul 02841; ${ }^{4}$ Department of Laboratory Medicine, \\ Yonsei University College of Medicine, Seoul 03722, Republic of Korea
}

Received September 1, 2016; Accepted September 11, 2017

DOI: $10.3892 /$ ijmm.2017.3142

\begin{abstract}
Mesenchymal stem cells (MSCs) differentiate into multiple lineages and are a promising source of cells for clinical use. Previously, we found that the gene distal-less homeobox 5 (DLX5) is specifically expressed in MSCs with osteogenic potential. Understanding the mechanism of osteogenesis is necessary for successful bone regeneration using MSCs. The aim of this study was to examine the function of the DLX5 gene in MSCs during osteogenesis (bone development). We analyzed the possible association between DLX 5 expression and osteogenesis-, chondrogenesis- and adipogenesis-related gene expression in different cells isolated from bone marrow and cord blood. Differentiation capacity was assessed by observing morphological changes, monitoring gene expression patterns, and staining with Von Kossa, safranin O, and Oil Red O. Suppression of DLX5 expression by means of a small interfering RNA (siRNA) downregulated osteogenic markers and reduced the signs of calcium mineralization. Tanshinone IIA is a known small molecule activator of bone morphogenetic protein $(B M P)$ signaling. Here, we report that induction of $D L X 5$ by tanshinone IIA in MSCs enhanced osteogenic differentiation. In addition, we showed that tanshinone IIA (as a mediator of BMP2 signaling) activates runt-related transcription factor 2 (RUNX2) in MSCs and initiates calcium mineralization during osteogenesis. Taken together, these findings indicate that, in MSCs, DLX5 is a
\end{abstract}

Correspondence to: Dr Hyun Ok Kim, Department of Laboratory Medicine, Yonsei University College of Medicine, 250 Seongsan-ro, Seodaemun-gu, Seoul, Republic of Korea

E-mail: hyunok1019@yuhs.ac

Dr Seung Gwan Lee, Department of Health and Environmental Science, College of Health Science, Korea University, Anam Campus, 145 Anam-ro, Seongbuk-gu, Republic of Korea

E-mail: seunggwan@korea.ac.kr

Key words: distal-less homeobox 5, mesenchymal stem cells, osteogenesis, tanshinone IIA master regulator of osteogenesis. Furthermore, tanshinone IIA may be valuable for stem cell-based therapies of certain bone diseases.

\section{Introduction}

Mesenchymal stem cells (MSCs) derived from various sources are valuable in regenerative medicine, including bone repair, because they can differentiate into multiple cell lineages, including osteoblasts, chondrocytes and adipocytes $(1,2)$. MSCs derived from different tissues have similar characteristics, but differ in their molecular profiles and differentiation potential (3).

Recently, MSCs have been applied to bone tissue engineering with a regenerative medicine approach (4). Osteogenic differentiation of MSCs is intricately regulated by multiple transcription factors and various cytokines and hormones (5-7). Previously, we found that distal-less homeobox 5 (DLX5), a homeodomain transcription factor encoded by a mammalian homolog of one of the Drosophila distal-less (DLL/DLX) genes that regulates the development of multiple cell types, is only expressed in MSCs with osteogenic potential (3). The discovery led us to examine whether DLX5 is critically involved in the differentiation of MSCs into osteoblasts.

Homeobox-containing genes play a key role as regulators of skeletal development (8). DLX genes that encode homeobox-containing transcription factors function in several developmental processes, including osteoblast development $(9,10)$. DLX5, which is involved in developing bone, cartilage, and teeth, is a member of the distal-less homeobox domain family (11-14). Overexpression of DLX5 is known to stimulate bone differentiation, and $D L X 5$-null mice exhibit abnormal osteogenesis (15-18). Although numerous studies strongly suggest that DLX5 is involved in osteogenesis, its functional role in this process is still obscure.

Here, we investigated the regulatory role of DLX5 in osteogenic differentiation of bone marrow- and cord blood-derived MSCs by examining the effects of DLX5 inhibition and the expression levels of osteogenesis-associated genes, including bone morphogenetic protein 2 (BMP2) and runt-related transcription factor 2 (RUNX2). BMP2 and RUNX2 play essential roles in bone development and maintenance by collaborating 
with other signaling molecules; however, they are insufficient to induce osteogenic differentiation $(19,20)$.

The aim of this study was to examine the key regulators of osteogenesis in MSCs. DLX5 is regulated by $B M P 2$, an inducer of osteogenesis $(21,22)$. To investigate the effects of DLX5 on osteogenic differentiation of MSCs, we examined osteogenic factors (DLX5 and RUNX2), chondrogenic factors [BMP7 and sex determining region Y-box 9 (SOX9)], and adipogenic factors [peroxisome proliferator-activated receptor $\gamma(P P A R G)$ and CCAAT-enhancer binding protein $\alpha(C / E B P A)]$. We demonstrated that the induction of $D L X 5$ led to osteoblast differentiation with the expression of several osteoblast markers, whereas the knockdown of DLX5 expression inhibited the osteogenesis of MSCs. Our data indicate that DLX5 is the master transcription factor stimulating the osteogenic factor $R U N X 2$ through $B M P 2$ signaling during osteogenesis.

Furthermore, we aimed to ascertain whether activation of DLX5 and/or BMP2 signaling by certain chemicals could induce osteogenic differentiation in MSCs. Tanshinone IIA is a major active phytochemical derived from phenanthrenequinone, which can be isolated from the roots of Salvia miltiorrhiza. It was found to enhance BMP2-stimulated differentiation of $\mathrm{C} 2 \mathrm{C} 12$ cells into osteoblasts via p38 activation (23). For the first time, we evaluated the effect of tanshinone IIA on the differentiation of MSCs into osteoblasts. This study demonstrated that tanshinone IIA affects osteogenesis from MSCs by augmenting DLX5.

These findings may be important for regenerative medicine, facilitating an increase in MSCs with osteogenic potential. Further, tanshinone IIA, as a small-molecule activator of $D L X 5$ and BMP signaling, could be one of the key molecules in $D L X 5$-induced osteogenesis of MSCs.

\section{Materials and methods}

Cells. Bone marrow and umbilical cord blood were collected from healthy donors after obtaining written informed consent. This study was approved by the Institutional Review Boards of Severance Hospital of Yonsei University Health System, Seoul, Korea.As previously described, mononuclearcells were isolated by Ficoll-Hypaque density gradient centrifugation (Pharmacia Biotech, Uppsala, Sweden) and the MSCs were cultured using the plastic adherence method (24). The cells were cultured at $37^{\circ} \mathrm{C}$ with $5 \% \mathrm{CO}_{2}$, and the medium [DMEM-low glucose supplemented with $10 \%$ fetal bovine serum (FBS) and $1 \%$ penicillin/streptomycin (P/S) (all from Invitrogen, Carlsbad, CA, USA)] was changed every 3 or 4 days. Tanshinone IIA from Sigma-Aldrich (St. Louis, MO, USA) was used for this study. During cultivation, cells were photographed under an inverted phase microscope (Olympus IX-71; Olympus, Tokyo, Japan) to compare morphologies.

Differentiation. To cause MSCs to differentiate into osteoblasts, chondrocytes, and adipocytes, bone marrow- and cord blood-derived MSCs were cultured in osteogenic induction medium, chondrogenic induction medium, and adipogenic induction medium for 3 weeks (Cambrex, Lonza, MD, USA). Osteoinductive medium-treated cells were used as the control. The medium was changed every 3 or 4 days, and the cells intended for chondrogenic differentiation were treated with
$10 \mathrm{ng} / \mathrm{ml}$ transforming growth factor (TGF)- $\beta 3$ (Cambrex) whenever the medium was replaced. For analysis, the induced cells were stained by Von Kossa to confirm osteogenesis, safranin $\mathrm{O}$ to confirm chondrogenesis, and Oil Red $\mathrm{O}$ to confirm adipogenesis. Images of the stained cells were captured using a phase microscope (Olympus IX-71; Olympus).

$R T$-PCR. Total RNA was extracted using TRIzol reagent, and standard reverse transcription (RT) was carried out using transcriptase II (both from Invitrogen). RT-PCR was performed using PCR primers (Bioneer, Daejeon, Korea) and annealing temperatures listed in Table I. Glyceraldehyde 3-phosphate dehydrogenase $(G A P D H)$ was used as an internal control. The signal intensity of the product was normalized to the respective GAPDH signal intensity. Osteoinductive medium-treated cells were used as control.

Small interfering RNA (siRNA) gene silencing. Specific knockdown of gene expression was performed using siRNA (Santa Cruz Biotechnology, Inc., Dallas, TX, USA) specific for DLX5. Briefly, 2x10 $10^{5}$ MSs were transfected with $10 \mu \mathrm{M}$ of negative control or targeted siRNA according to the manufacturer's protocol. Following incubation for $7 \mathrm{~h}$ at $37^{\circ} \mathrm{C}$ and $5 \% \mathrm{CO}_{2}$, normal growth medium was added. After one day, medium was replaced with fresh normal growth medium. The effect of gene knockdown by siRNA was evaluated by RT-PCR assay. MSCs were treated with DLX5-siRNA when medium was replaced for the entire induction period.

Cell viability test. The viability of chemically treated cells was analyzed by the trypan blue exclusion method (Invitrogen). Briefly, cells were seeded at a density of $5 \times 10^{4}$ cells in 12-well plates (Nunc, Roskilde, Denmark). The next day, 3, 6, 12, 24 or $48 \mu \mathrm{M}$ of tanshinone IIA was added to the cells. After 3 days, the cells were harvested and trypan blue-stained cells were counted.

Analysis of calcium concentration. Following osteogenic induction, the calcium content of cells was determined using a Calcium (CPC) LiquiColor Test (Stanbio Laboratory, Boerne, TX, USA) according to the manufacturer's instructions. Briefly, the cells were washed with phosphate-buffered saline (PBS; Invitrogen) and $0.5 \mathrm{~N} \mathrm{HCl}$ was added to the cells. The cells were harvested and transferred to a new tube. After shaking for $3 \mathrm{~h}$ with an orbital shaker, the supernatant was transferred to a new tube for analysis. Color and base reagents were added to the supernatant, and then absorbances were detected at $550 \mathrm{~nm}$. The cells cultured in DMEM were used as the control.

Statistical analysis. Quantitative data are expressed as the means \pm standard deviation (SD). Statistical comparisons were performed by a Student's t-test and one-way analysis of variance (ANOVA) with post-hoc Bonferroni corrections. The differences were considered statistically significant at $\mathrm{P}<0.05$.

\section{Results}

Characterization of bone marrow-and cord blood-derived MSCs. All MSCs derived from bone marrow and cord blood 
Table I. Primer sequences.

\begin{tabular}{|c|c|c|c|}
\hline $\begin{array}{l}\text { Gene } \\
\text { name }\end{array}$ & $\begin{array}{l}\text { Primer sequences } \\
\qquad\left(5^{\prime}-3^{\prime}\right)\end{array}$ & $\begin{array}{c}\text { Annealing } \\
\text { temperature }\left({ }^{\circ} \mathrm{C}\right)\end{array}$ & $\begin{array}{l}\text { Product } \\
\text { size (bp) }\end{array}$ \\
\hline$B M P 2$ & $\begin{array}{l}\text { Forward: CGAGGTCCTGAGCGAGTTCGAG } \\
\text { Reverse: TGGCAGTAAAAGGCGTGATACC }\end{array}$ & 60 & 838 \\
\hline RUNX2 & $\begin{array}{l}\text { Forward: GACCAGTCTTACCCСТCCTACC } \\
\text { Reverse: CTGCCTGGCTCTTCTTACTGAG }\end{array}$ & 58 & 190 \\
\hline$D L X 5$ & $\begin{array}{l}\text { Forward: ACCATCCGTCTCAGGAATCG } \\
\text { Reverse: ACCTTCTCTGTAATGCGGCC }\end{array}$ & 60 & 384 \\
\hline GAPDH & $\begin{array}{l}\text { Forward: GTGGTCTCCTCTGACTTCAACA } \\
\text { Reverse: CTCTTCCTCTTGTGCTCTTGCT }\end{array}$ & 62 & 210 \\
\hline$B M P 7$ & $\begin{array}{l}\text { Forward: CCAACGTCATCCTGAAGAAATAC } \\
\text { Reverse: GCTTGTAGGATCTTGTTCATTGG }\end{array}$ & 60 & 271 \\
\hline SOX9 & $\begin{array}{l}\text { Forward: GCCGGGCAAGGCTGACCTGAAG } \\
\text { Reverse: TTCTGGTGGTCGGTGTAGTCGT }\end{array}$ & 62 & 605 \\
\hline PPARG & $\begin{array}{l}\text { Forward: TCTCTCCGTAATGGAAGACC } \\
\text { Reverse: GCATTATGAGACATCCCCAC }\end{array}$ & 55 & 474 \\
\hline C/EBPA & $\begin{array}{l}\text { Forward: CCAAGAAGTCGGTGGACAAGAA } \\
\text { Reverse: TCATTGTCACTGGTCAGCTCCA }\end{array}$ & 62 & 145 \\
\hline Osterix & $\begin{array}{l}\text { Forward: TAATGGGCTCCTTTCACCTG } \\
\text { Reverse: CACTGGGCAGACAGTCAGAA }\end{array}$ & 60 & 161 \\
\hline Osteopontin & $\begin{array}{l}\text { Forward: GAGACCCTTCCAAGTAAGTCCA } \\
\text { Reverse: GATGTCCTCGTCTGTAGCATCA }\end{array}$ & 62 & 354 \\
\hline Type I collagen & $\begin{array}{l}\text { Forward: CACAGAGGTTTCAGTGGTTTGG } \\
\text { Reverse: GCACCAGTAGCACCATCATTTC }\end{array}$ & 62 & 191 \\
\hline$A P 2$ & $\begin{array}{l}\text { Forward: AAGAAGTAGGAGTGGGCTTTGC } \\
\text { Reverse: CCACCACCAGTTTATCATCCTC }\end{array}$ & 62 & 381 \\
\hline
\end{tabular}

BMP2, bone morphogenetic protein; RUNX2, runt-related transcription factor 2; DLX5, distal-less homeobox 5; GAPDH, glyceraldehyde 3-phosphate dehydrogenase; SOX9, sex determining region Y-box 9; PPARG, peroxisome proliferator-activated receptor $\gamma$; C/EBPA, CCAAT-enhancer binding protein $\alpha$.

showed a similar spindle-shaped morphology (Fig. 1A). Surface markers of the MSCs were analyzed, and the results showed that all cells exhibited similar immunophenotypic patterns. The cells were positive for CD29, CD44, CD73, CD90 and CD105, all known markers of MSCs, whereas the MSCs were negative for markers of endothelial and hematopoietic cells such as CD14, CD31, CD34, CD45 and CD106 (data not shown). These results confirmed that the cultured cells expressed typical MSC surface markers. To determine their differentiation capacity, the cells were induced to display osteogenic, chondrogenic, or adipogenic phenotypes. Of the MSCs derived from bone marrow and cord blood, one sample of cord blood MSCs (CB-MSC1) did not differentiate into osteoblasts despite a sufficient induction period, whereas the other MSCs exhibited tri-lineage differentiation potential, developing into osteoblasts, chondrocytes and adipocytes (Fig. 1B). Together, these data indicate that not all MSCs with fibroblast-like morphologies and MSC surface proteins have tri-lineage differentiation capacities.
Osteogenesis and DLX5 expression of MSCs. To investigate osteogenic molecular profiles associated with morphological changes, we performed an RT-PCR analysis of specific osteogenesis markers, namely $B M P 2, R U N X 2$ and $D L X 5$, during the induction of bone marrow- and cord blood-derived MSCs from different donors. BMP2, RUNX2 and DLX 5 were expressed in all MSCs that differentiated into osteoblasts, regardless of the induction period (Fig. 2). However, CB-MSC1, which did not differentiate into an osteogenic phenotype, did not express $B M P 2$ and $D L X 5$ at any time in the induction environment. Interestingly, RUNX2 was independently expressed in all MSCs, regardless of their osteogenic potential (Fig. 2). These results coincide with previous data, confirming $D L X 5$ as a marker for the osteogenic potential of MSCs (3). Based on these results, we noted that $D L X 5$ with $B M P 2$ signaling may be the only critical factors for osteogenesis of MSCs.

Effect of DLX5 knockdown on the differentiation potential of MSCs. To examine the role of DLX5 in the tri-lineage 


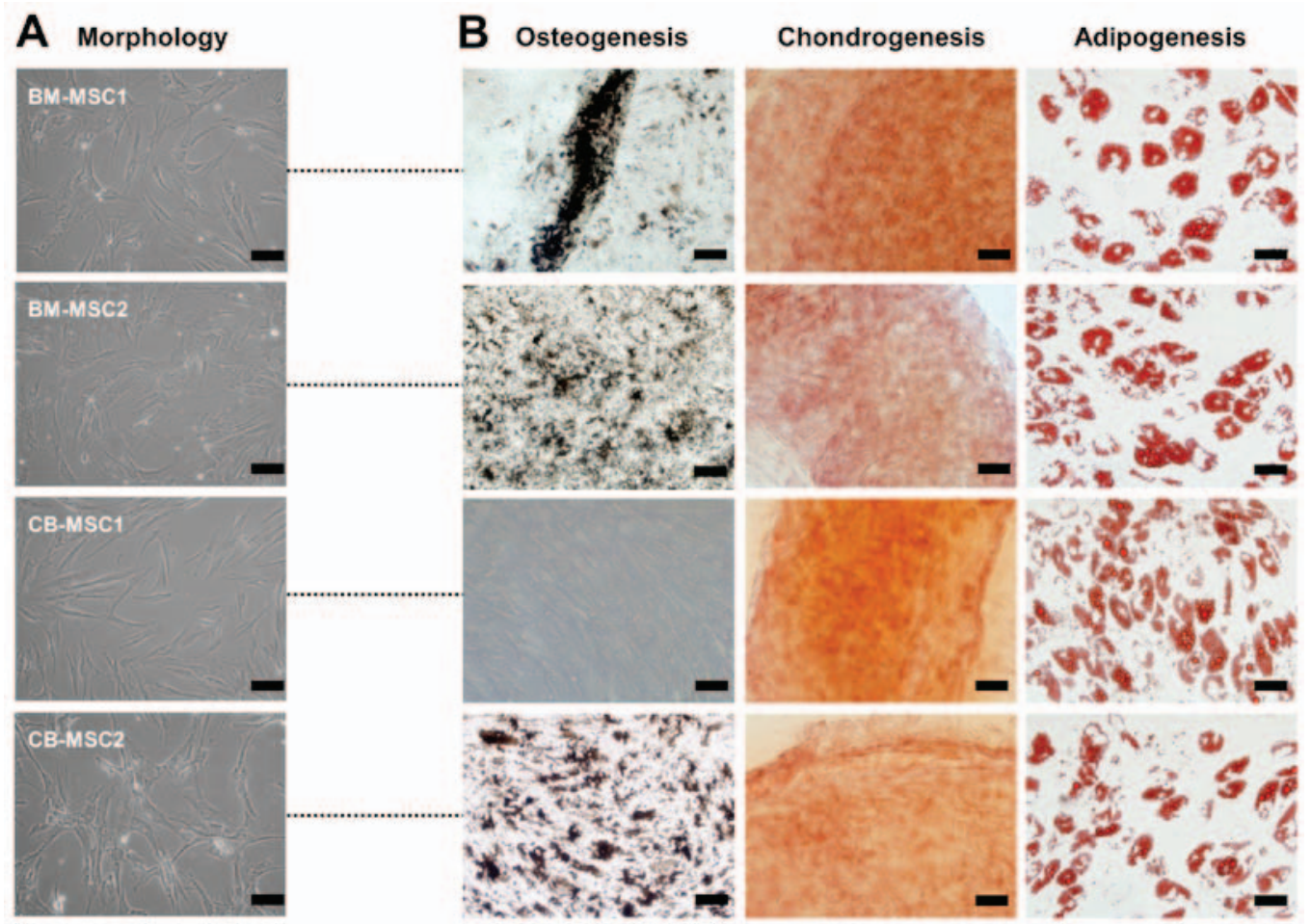

Figure 1. Morphology and differentiation potential of mesenchymal stem cells (MSCs). (A) Phase contrast images of MSCs derived from bone marrow and cord blood (scale bar, $100 \mu \mathrm{m}$; magnification, x200). (B) Differentiation capacity was examined after induction. Osteogenesis was verified by Von Kossa staining (scale bar, $100 \mu \mathrm{m}$; magnification, x200). Chondrogenesis was investigated by safranin O staining (scale bar, $100 \mu \mathrm{m}$; magnification, x200). Adipogenesis was examined by Oil Red O staining (scale bar, $50 \mu \mathrm{m}$; magnification, $\mathrm{x} 400$ ).
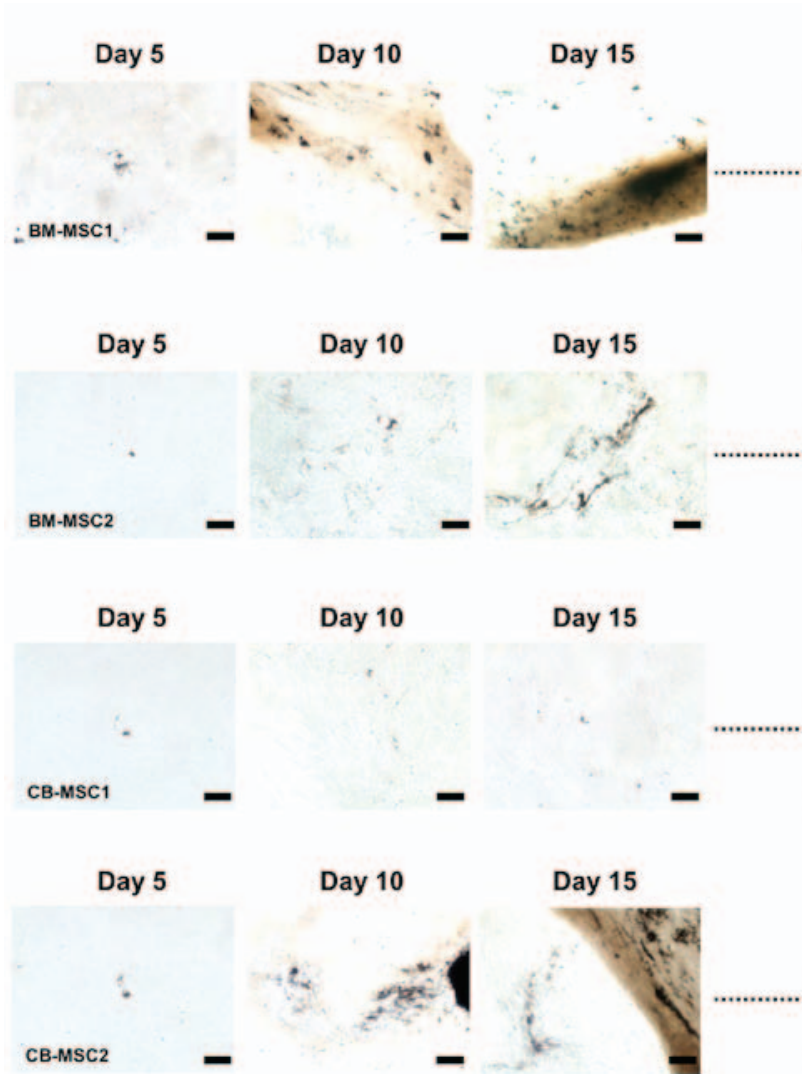

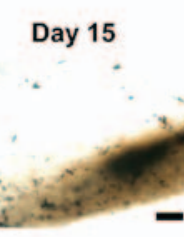

Day 15
Day 10

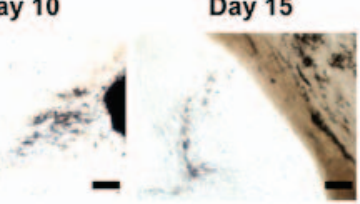

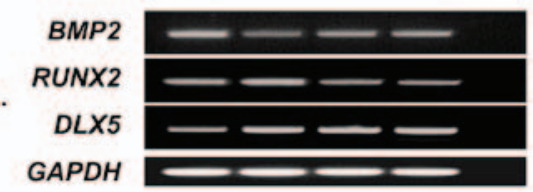

GAPDH

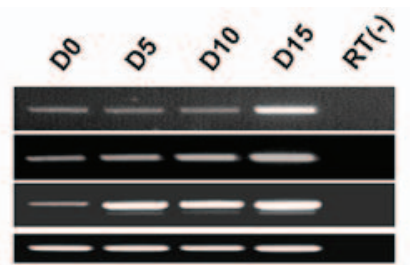

GAPDH

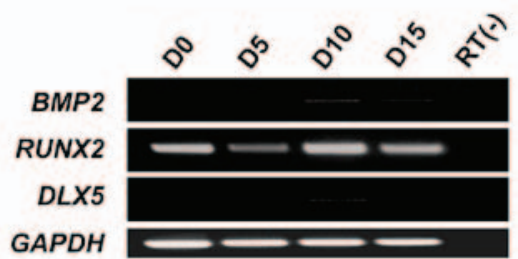

Figure 2. Time course images of osteogenic differentiation and expression of osteogenesis-related markers. Top panels: images of BM-MSC1 induced by osteogenic medium, and RT-PCR analysis of osteogenesis-associated markers in BM-MSC1. Second row: images of BM-MSC2 differentiated by osteogenic medium, and RT-PCR analysis of osteogenesis-related markers in BM-MSC2. Third row: images of CB-MSC1 induced by osteogenic medium, and RT-PCR analysis of osteogenesis-associated markers in CB-MSC1. Lower panels: images of CB-MSC2 induced by osteogenic medium, and RT-PCR analysis of osteogenesis-related markers in CB-MSC2. Osteogenesis was evaluated by Von Kossa staining (scale bar, $100 \mu \mathrm{m}$; magnification, x200). 

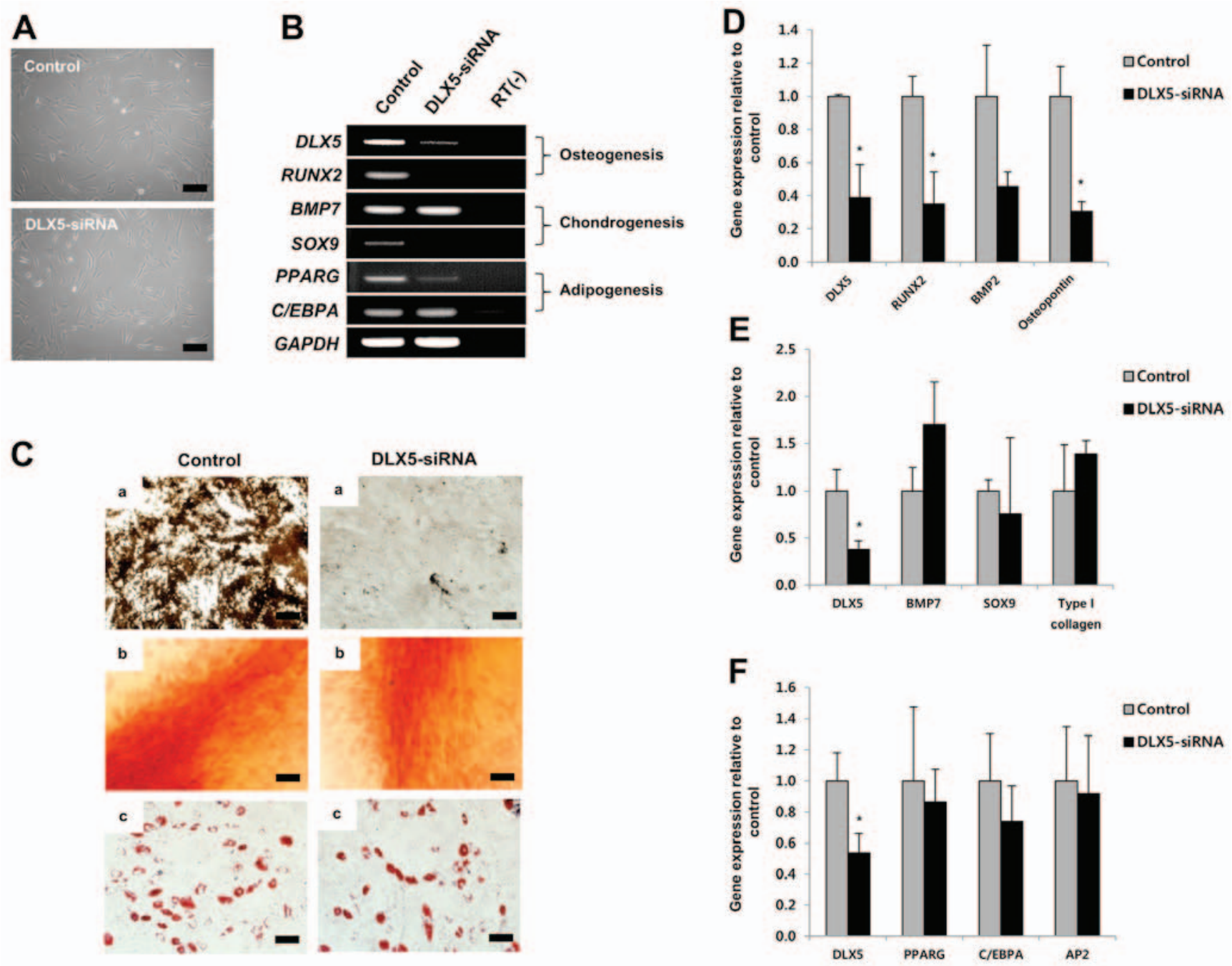

Figure 3. Changes in mesenchymal stem cells (MSCs) following knockdown of distal-less homeobox 5 (DLX5). (A) Phase contrast images before and after silencing of DLX5 (scale bar, $200 \mu \mathrm{m}$; magnification, x100). (B) RT-PCR analysis of osteogenesis-, adipogenesis- and chondrogenesis-associated genes in the control and DLX5 siRNA-transfected MSCs. Decreased osteogenic differentiation of MSCs following knockdown of $D L X 5$. (C) Inhibition of $D L X 5$ expression prevents osteogenesis of MSCs. The differentiation capacity of MSCs was analyzed after induction. (a) Osteogenesis assessed by Von Kossa staining. (b) Chondrogenesis assessed by safranin O staining. (c) Adipogenesis assessed by Oil Red O staining (scale bar, $100 \mu \mathrm{m}$ ). Relative mRNA expression levels of differentiation-associated markers in the control and DLX5-siRNA-transfected MSCs after (D) osteogenic induction, (E) chondrogenic induction, and (F) adipogenic induction. ${ }^{\prime} \mathrm{P}<0.05$.

differentiation of MSCs, we employed siRNA-mediated knockdown of $D L X 5$, using $D L X 5$-expressing cells. The morphologies of cultured MSCs before induction were unaffected, compared to those of the control, by short-term treatment with $D L X 5$-siRNA (Fig. 3A). RT-PCR results showed that $D L X 5$-siRNA substantially decreased expression of the DLX5 gene and completely silenced the osteogenic marker gene RUNX2 and chondrogenic marker gene SOX9. $C / E B P A$ of the adipogenic marker genes was unaffected by $D L X 5$-siRNA treatment, while PPARG expression was slightly decreased (Fig. 3B). These results indicate that osteogenesis of MSCs can be markedly affected by $D L X 5$-siRNA knockdown.

We next performed a differentiation assay in the presence of DLX5-siRNA. Surprisingly, the osteogenic capacity of MSCs treated with $D L X 5$-siRNA was significantly decreased, whereas chondrogenic and adipogenic capacities were similar, relative to that of the control, although MSCs did not express the SOX9 gene following DLX5-siRNA treatment (Fig. 3C). We then analyzed gene expression levels related to tri-lineage differentiation by RT-PCR after induction. Expression of the following genes was evaluated: DLX5, RUNX2, BMP2 and osteopontin for osteogenesis; DLX5, BMP7, SOX9 and type I collagen for chondrogenesis; and DLX5, PPARG, C/EBPA and $A P 2$ for adipogenesis. The levels of RUNX2 and osteopontin gene expression were significantly decreased relative to the control by inhibition of $D L X 5$ (Fig. 3D), whereas no significant differences were detected in the expression of chondrogenesis- (Fig. 3E) or adipogenesis- (Fig. 3F) related genes. Relative gene expression was normalized to that of $G A P D H$, the internal control. These results strongly suggest that $D L X 5$ is the most powerful and specific transcription factor for osteogenic differentiation.

Tanshinone IIA induces DLX5 through BMP2 signaling in MSCs. Tanshinone IIA, a major active phytochemical, is involved in bone metabolism. It has a wide range of biological activities, including anti-inflammation and antioxidation (25-27). Moreover, tanshinone IIA is known to 
A
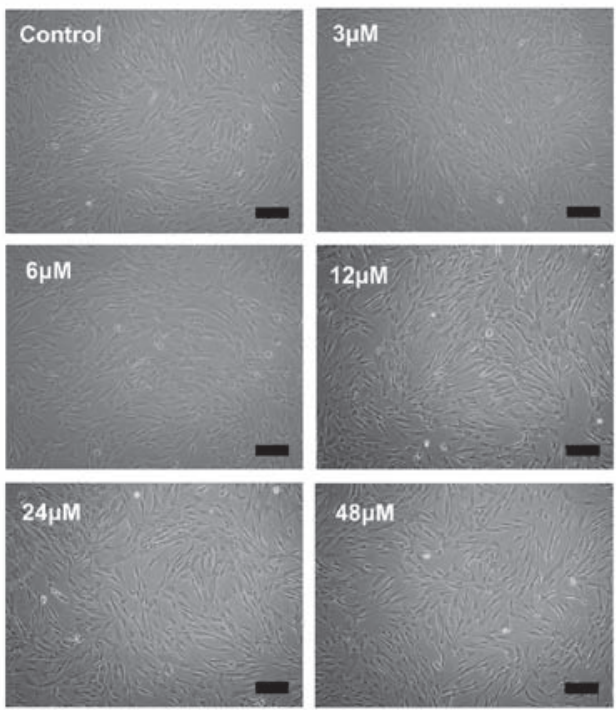

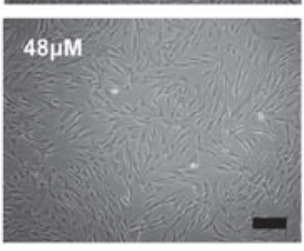

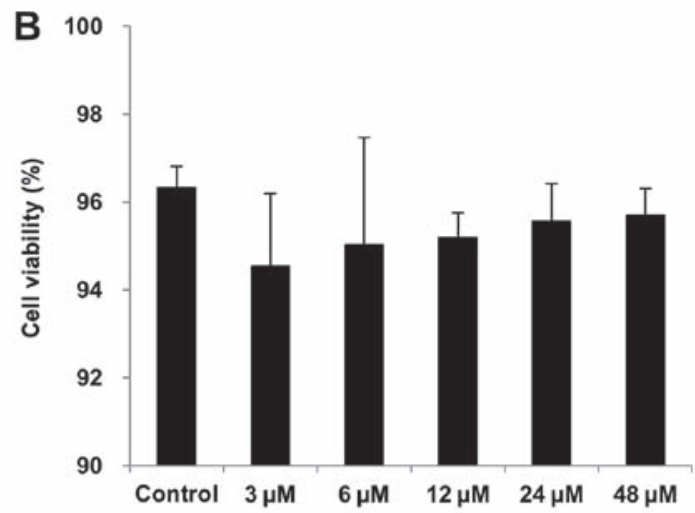

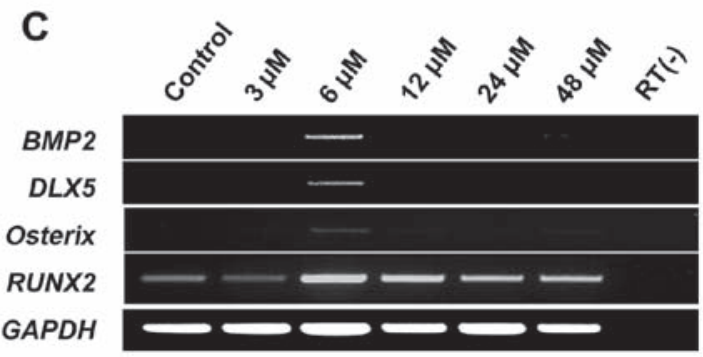

Figure 4. Effect of tanshinone IIA on osteogenesis. (A) Mesenchymal stem cells (MSCs) treated with tanshinone IIA were photographed after 3 days (scale bar, $200 \mu \mathrm{m}$; magnification, x100). (B) Cell viability was determined by trypan blue staining. The cell viability of the tanshinone IIA-treated MSCs was $>90 \%$. (C) Induction of osteogenesis-associated genes by tanshinone IIA treatment. Treatment only with $6 \mu$ M tanshinone IIA induced $B M P 2$, distal-less homeobox 5 (DLX5) and osterix genes.

enhance BMP-2 stimulation of cells to differentiate into osteoblasts (23). Ultimately, stimulation by tanshinone IIA induces osteogenesis via regulation of osteogenic factors, including $B M P 2$ and $D L X 5$.

Therefore, we tested whether tanshinone IIA could induce DLX 5 in DLX5 not-expressing MSCs. Cell morphologies were not affected by $3,6,12,24$ or $48 \mu \mathrm{M}$ of tanshinone IIA treatment (Fig. 4A). In addition, cell viability of $>90 \%$ was maintained with tanshinone IIA treatment (Fig. 4B). Remarkably, BMP2, DLX5 and osterix genes were only induced in response to $6 \mu \mathrm{M}$ of tanshinone IIA treatment, as early as after 3 days of cultivation, indicating the activation of $D L X 5$ by the $B M P 2$ pathway. However, $R U N X 2$ was similarly expressed (Fig. 4C). Taken together, these results show that tanshinone IIA can induce DLX5, as well as the most prominent factors of osteogenesis.

Tanshinone IIA enhances osteogenesis of MSCs by inducing DLX5 with BMP2. We next treated DLX5 not-expressing MSCs with $6 \mu \mathrm{M}$ tanshinone IIA to induce osteogenesis. After tanshinone IIA treatment, we did not find any differences in our morphological investigation. Subsequently, the effect of tanshinone IIA on the osteogenic potential of MSCs was evaluated by analyzing the expression levels of genes associated with osteogenic differentiation and by visualizing the staining of induced cells. Tanshinone IIA significantly induced BMP2 and $D L X 5$, as well as upregulated $R U N X 2$ genes involved in osteogenesis. The expression of $S O X 9$, which is involved in chondrogenesis, was similarly upregulated, compared to that of the control, despite tanshinone IIA treatment, whereas $B M P 7$ expression was decreased (Fig. 5A). Interestingly, expression of $C / E B P A$, a transcription factor for adipogenesis, was completely inhibited by tanshinone IIA (Fig. 5A). The differentiation assay revealed that tanshinone IIA specifically enhanced osteogenesis of MSCs (Fig. 5B). This finding was confirmed by calcium deposition assay, as shown in Fig. 5C. Furthermore, tanshinone IIA-mediated enhancement of $D L X 5$ through the induction of $B M P 2$ upregulated mRNA expression of RUNX2 and osteopontin during osteogenic differentiation of MSCs (Fig. 5D). As shown in Fig. 5A, we again confirmed that the expression of $B M P 7$ was decreased significantly during chondrogenesis (Fig. 5E), and that $C / E B P A$ expression was suppressed by tanshinone IIA during adipogenic differentiation (Fig. 5F). Relative gene expression in the differentiated MSCs was normalized to $G A P D H$, the internal control. Surprisingly, tanshinone IIA-treated MSCs differentiated into chondrocytes and adipocytes despite the suppression of BMP7 and C/EBPA genes (Fig. 5B). Taken together, these results indicate that tanshinone IIA induces osteogenesis in $D L X 5$ not-expressing MSCs by activating $D L X 5$ through $B M P 2$ expression.

\section{Discussion}

MSCs derived from various tissues have become a preferred cell type in the field of regenerative medicine due to their plastic and immunosuppressive properties (28). Although stem cells hold great promise for future therapeutic applications, clinical applications using these cells have been stymied by an insufficient understanding of stem cell biology, including the complex genetic processes in these cells. Therefore, further characterization of stem cells via diverse approaches such as genomics and proteomics will be critical for a better understanding and utilization of stem cells. 
A

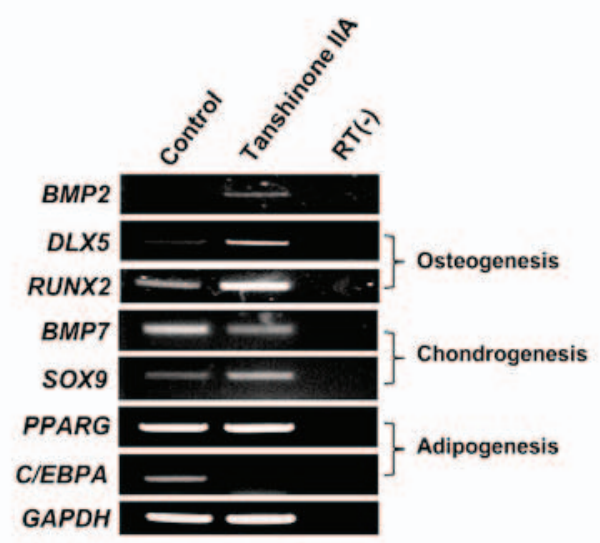

B

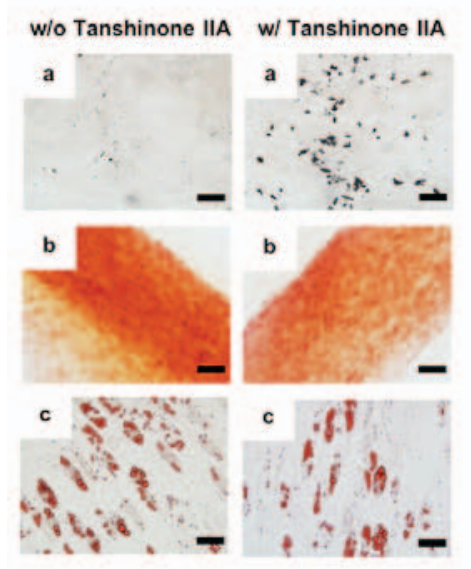

C

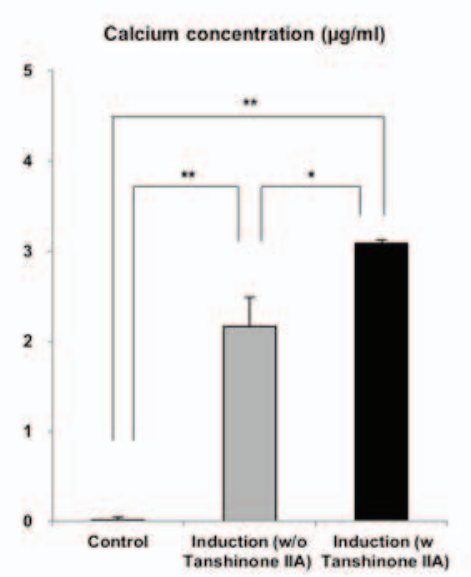

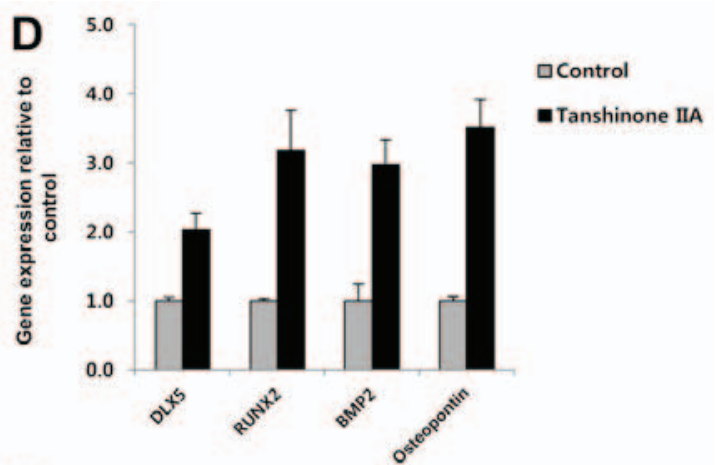
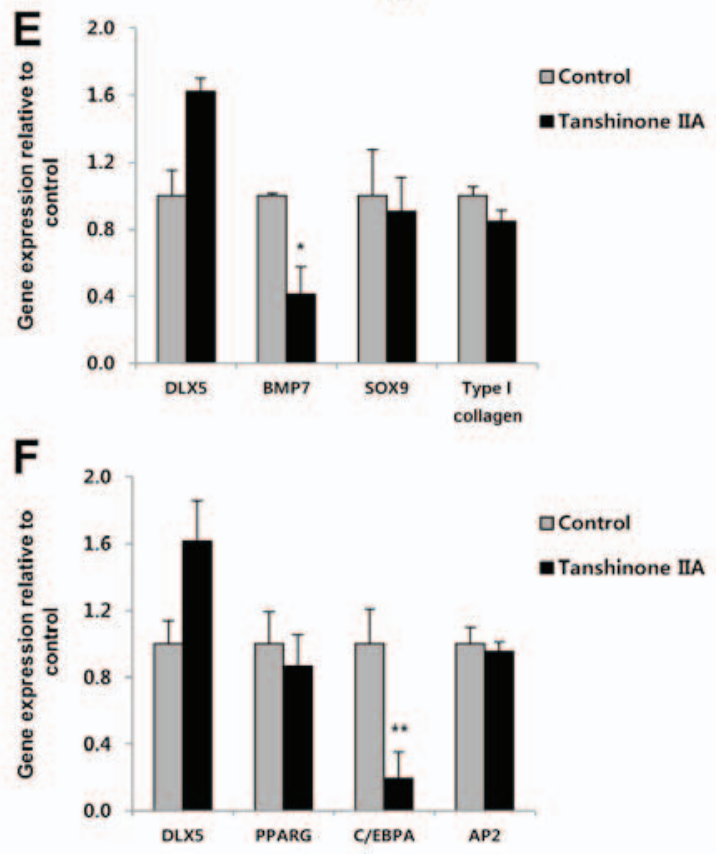

Figure 5. Changes in mesenchymal stem cells (MSCs) following tanshinone IIA treatment. (A) RT-PCR analysis of osteogenesis-, chondrogenesis- and adipogenesis-associated genes in the control and tanshinone IIA-induced MSCs. Increased osteogenic differentiation of MSCs by tanshinone IIA. (B) Induction of distal-less homeobox 5 (DLX5) expression enhanced osteogenesis of $D L X 5$ not-expressing MSCs. The differentiation capacity of the cells was analyzed after induction. (a) Osteogenesis assessed by Von Kossa staining (scale bar, $50 \mu \mathrm{m}$ ). (b) Chondrogenesis assessed by safranin $\mathrm{O}$ staining (scale bar, $100 \mu \mathrm{m}$ ). (c) Adipogenesis assessed by Oil Red O staining (scale bar, $50 \mu \mathrm{m}$ ). (C) Calcium concentrations were measured in triplicate using a Calcium LiquiColor test. Relative mRNA expression levels of differentiation-associated markers in the control and DLX5-induced MSCs after (D) osteogenic induction, (E) chondrogenic induction, and (F) adipogenic induction. ${ }^{*} \mathrm{P}<0.05$ and ${ }^{* *} \mathrm{P}<0.01$.

Previous research on MSCs from different sources has documented their variable differentiation potential and has shown that this variation in osteogenic potential depends on DLX 5 gene expression (3). Consistent with the results of a previous study, DLX5 expression was not detected in MSCs that did not have the capacity to differentiate into osteoblasts. In addition, $B M P 2$ gene expression was not observed in this study when DLX5 not-expressing MSCs were maintained in an osteogenic environment. Differentiation of stem cells is a complex process governed by various genetic networks, and the biological functions of genes associated with MSC differentiation remain unclear. In the present study, we aimed to investigate the precise role of the DLX5 gene during the osteogenesis of MSCs, including whether the DLX5 gene is important for initiating osteogenesis and whether the gene is sufficient to completely drive osteogenesis from MSCs.

$D L X 5$, a member of the DLX family of homeobox genes, is known to be a key regulator of differentiation involved in developing skeletal elements and of osteogenesis and chondrogenesis in the formation of hard tissues (29). Several studies suggest that $D L X 5$ acts as a modulator of osteogenesis in various cell types $(18,30)$. However, the mechanism underlying osteogenic differentiation, including the role of $D L X 5$, is still controversial, especially in MSCs. In this study, we used MSCs derived from bone marrow and cord blood, less than 5 passages, and expressing and/or not expressing the $D L X 5$ gene to identify the effects, including the effects on genes activated and inactivated by $D L X 5$ in the course of differentiation. In order to investigate the role of $D L X 5$, we profiled morphological and gene expression changes associated with osteogenesis of MSCs. As mentioned above, DLX5 not-expressing MSCs without BMP2 expression failed to differentiate into osteoblasts. However, $R U N X 2$ was consistently expressed during osteogenic induction, irrespective of the expression of $B M P 2$ and $D L X 5$. To further examine the effect of $D L X 5$ on osteogenesis, siRNA, which targets the DLX5 gene, was used to inhibit endogenous $D L X 5$ expression in MSCs. Knockdown of DLX 5 using siRNA did not alter the morphology and the proliferation rate of the cells. Seventy-two hours after siRNA transfection, RUNX2 and SOX9 genes specific for osteogenesis and chondrogenesis, 


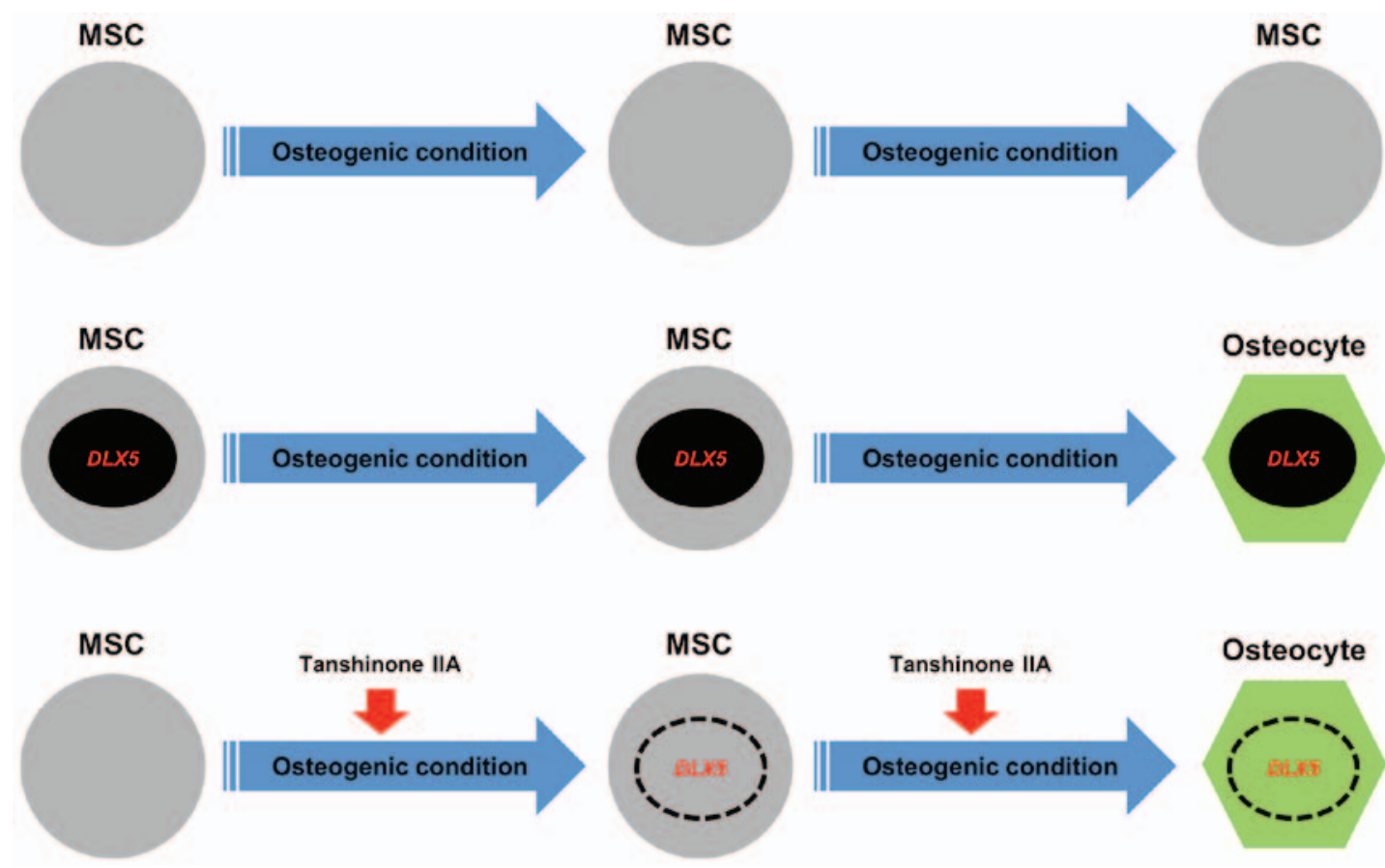

Figure 6. Model summarizing osteogenesis in mesenchymal stem cells (MSCs). Distal-less homeobox 5 (DLX5) and DLX5 inducer, tanshinone IIA, promote the differentiation of MSCs into osteoblasts by upregulating osteogenesis-related genes.

respectively, were inhibited in the cultured cells. In addition, osteogenic differentiation of MSCs was significantly suppressed by DLX5-siRNA, with a decrease in osteopontin gene expression compared to the control. In contrast, the chondrogenic and adipogenic potential of these cells was unaffected by $D L X 5$-siRNA, as proteoglycans for chondrogenesis and neutral lipids for adipogenesis were similarly detected by immunohistochemical staining in control cells exposed to inductive conditions. These results indicate that $D L X 5$ drives the osteogenic differentiation program in MSCs.

Tanshinone IIA is a major active phytochemical that is isolated from the roots of $S$. miltiorrhiza and enhances BMP2-stimulated differentiation of myoblasts into osteoblasts (23). However, there is little research on the effects of tanshinone IIA on the osteogenic differentiation of MSCs. To examine the effects of tanshinone IIA on MSCs, DLX5 not-expressing cells were cultured with tanshinone IIA. Our data showed that $D L X 5$ induced by tanshinone IIA activated osteogenic marker genes, including osterix, RUNX2 and osteopontin, in cooperation with $B M P 2$, with cell morphologies that remained similar to control cells, whereas tanshinone IIA suppressed BMP7 gene (chondrogenesis) and C/EBPA gene (adipogenesis) expression. These results are in line with previous studies showing that $D L X 5$ plays a role in $B M P 2$-induced osteogenesis through upregulation of the RUNX2 gene, and that it functions as part of the BMP signaling pathway $(21,31)$. In addition, these results suggest that tanshinone IIA is involved in the $B M P 2$ signaling pathway and $D L X 5$-induced osteogenic differentiation. Functional validation with tanshinone IIA was carried out by differentiation assays and PCR analysis. MSCs with strongly upregulated $D L X 5, R U N X 2, B M P 2$ and osteopontin genes following tanshinone IIA treatment differentiated into osteoblasts and showed significantly increased calcium deposition compared to DLX5 not-expressing cells. However, a higher concentration of tanshinone IIA $(6 \mu \mathrm{M})$ decreased the osteogenic capacity of MSCs, indicating that osteogenic differentiation following DLX5 induction in treated cells is tanshinone IIA concentration-dependent (data not shown). Additionally, MSCs treated with tanshinone IIA differentiated into chondrocytes and adipocytes despite inhibition of $B M P 7$ and $C / E B P A$, indicating that these genes may not be essential factors for differentiation. Furthermore, DLX 5 may play a role as an osteogenesis determinant through the upregulation of $R U N X 2$ and the downregulation of $B M P 7$ and C/EBPA.

Here, we showed that $D L X 5$ is a specific target of $B M P 2$-induced osteogenesis of MSCs, demonstrating that $D L X 5$ and $B M P 2$ can contribute to RUNX2-independent regulation of osteogenesis. This indicates that $R U N X 2$ induction is not mediated by $B M P 2$ and $D L X 5$ in MSCs as previously reported (32). Additionally, we confirmed that RUNX2 is not essential for the induction of an osteogenic lineage of MSCs, indicating that $R U N X 2$ may function in concert with $D L X 5$ to induce osteogenic differentiation by regulating the expression of osteogenesis-specific markers such as osteopontin. These findings are in agreement with previous results showing that $D L X 5$ plays an important role in the activation of osteogenesis by regulating BMP-induced RUNX2 (22). Moreover, we showed that tanshinone IIA is capable of stimulating $D L X 5$ expression with $B M P 2$, resulting in osteogenic differentiation of MSCs. To the best of our knowledge, we showed for the first time that tanshinone IIA can be used in place of $D L X 5$ to induce differentiate of MSCs into osteoblasts. Fig. 6 shows a schematic model that summarizes the osteogenesis of MSCs by the induction of the DLX5 gene using tanshinone IIA. Our findings contribute to the development of effective bone regeneration therapies for the treatment of bone diseases. Furthermore, tanshinone IIA is a chemical compound that may be used for the treatment of bone diseases; however, 
our in vitro results require in vivo validation. Additional investigations are required for a deeper understanding of the upstream and downstream signaling pathways related to other osteogenesis-related factors.

In conclusion, our data showed that $D L X 5$ plays a role as a master transcription factor in osteogenic differentiation, and that tanshinone IIA, coincident with the induction of $B M P 2$, synergistically induces osteogenesis by targeting $D L X 5$.

\section{Acknowledgements}

The present study was supported by Grant HI15C0942 from the Korea Health Technology R\&D Project through the Korea Health Industry Development Institute (KHIDI), funded by the Ministry of Health and Welfare, Republic of Korea.

\section{References}

1. Koç ON and Lazarus HM: Mesenchymal stem cells: Heading into the clinic. Bone Marrow Transplant 27: 235-239, 2001.

2. Prockop DJ, Gregory CA and Spees JL: One strategy for cell and gene therapy: Harnessing the power of adult stem cells to repair tissues. Proc Natl Acad Sci USA 100 (Suppl 1): 11917-11923, 2003.

3. Heo JS, Choi Y, Kim HS and Kim HO: Comparison of molecular profiles of human mesenchymal stem cells derived from bone marrow, umbilical cord blood, placenta and adipose tissue. Int J Mol Med 37: 115-125, 2016.

4. Meijer GJ, de Bruijn JD, Koole R and van Blitterswijk CA: Cell-based bone tissue engineering. PLoS Med 4: e9, 2007.

5. Komori T: Regulation of osteoblast differentiation by transcription factors. J Cell Biochem 99: 1233-1239, 2006.

6. Wagner ER, Luther G, Zhu G, Luo Q, Shi Q, Kim SH, Gao JL, Huang E, Gao Y, Yang K, et al: Defective osteogenic differentiation in the development of osteosarcoma. Sarcoma 2011: 325238, 2011.

7. Yamaguchi A, Komori T and Suda T: Regulation of osteoblast differentiation mediated by bone morphogenetic proteins, hedgehogs, and Cbfa1. Endocr Rev 21: 393-411, 2000.

8. Chen X, Li X, Wang W and Lufkin T: Dlx5 and Dlx6: An evolutionary conserved pair of murine homeobox genes expressed in the embryonic skeleton. Ann NY Acad Sci 785: 38-47, 1996.

9. Depew MJ, Lufkin T and Rubenstein JL: Specification of jaw subdivisions by Dlx genes. Science 298: 381-385, 2002.

10. Robledo RF, Rajan L, Li X and Lufkin T: The Dlx5 and Dlx6 homeobox genes are essential for craniofacial, axial, and appendicular skeletal development. Genes Dev 16: 1089-1101, 2002.

11. Ferrari D, Sumoy L, Gannon J, Sun H, Brown AM, Upholt WB and Kosher RA: The expression pattern of the Distal-less homeobox-containing gene Dlx-5 in the developing chick limb bud suggests its involvement in apical ectodermal ridge activity, pattern formation, and cartilage differentiation. Mech Dev 52: 257-264, 1995.

12. Newberry EP, Latifi T and Towler DA: Reciprocal regulation of osteocalcin transcription by the homeodomain proteins Msx2 and Dlx 5. Biochemistry 37: 16360-16368, 1998.

13. Ryoo HM, Hoffmann HM, Beumer T, Frenkel B, Towler DA, Stein GS, Stein JL, van Wijnen AJ and Lian JB: Stage-specific expression of Dlx-5 during osteoblast differentiation: Involvement in regulation of osteocalcin gene expression. Mol Endocrinol 11: 1681-1694, 1997

14. Weiss KM, Ruddle FH and Bollekens J: Dlx and other homeobox genes in the morphological development of the dentition. Connect Tissue Res 32: 35-40, 1995.
15. Acampora D, Merlo GR, Paleari L, Zerega B, Postiglione MP, Mantero S, Bober E, Barbieri O, Simeone A and Levi G: Craniofacial, vestibular and bone defects in mice lacking the Distal-less-related gene Dlx5. Development 126: 3795-3809, 1999.

16. Depew MJ,Liu JK, Long JE, Presley R, Meneses JJ, Pedersen RA and Rubenstein JL: Dlx5 regulates regional development of the branchial arches and sensory capsules. Development 126: 3831-3846, 1999.

17. Miyama K, Yamada G, Yamamoto TS, Takagi C, Miyado K, Sakai M, Ueno N and Shibuya H: A BMP-inducible gene, dlx5, regulates osteoblast differentiation and mesoderm induction. Dev Biol 208: 123-133, 1999.

18. Tadic T, Dodig M, Erceg I, Marijanovic I, Mina M, Kalajzic Z, Velonis D, Kronenberg MS, Kosher RA, Ferrari D, et al: Overexpression of Dlx 5 in chicken calvarial cells accelerates osteoblastic differentiation. J Bone Miner Res 17: 1008-1014, 2002.

19. Maeno T, Moriishi T, Yoshida CA, Komori H, Kanatani N, Izumi S, Takaoka K and Komori T: Early onset of Runx2 expression caused craniosynostosis, ectopic bone formation, and limb defects. Bone 49: 673-682, 2011.

20. Rosen V: BMP2 signaling in bone development and repair. Cytokine Growth Factor Rev 20: 475-480, 2009.

21. Holleville N, Quilhac A, Bontoux M and Monsoro-Burq AH: BMP signals regulate Dlx 5 during early avian skull development. Dev Biol 257: 177-189, 2003.

22. Lee MH, Kim YJ, Kim HJ, Park HD, Kang AR, Kyung HM, Sung JH, Wozney JM, Kim HJ and Ryoo HM: BMP-2-induced Runx2 expression is mediated by Dlx5, and TGF-beta 1 opposes the BMP-2-induced osteoblast differentiation by suppression of Dlx5 expression. J Biol Chem 278: 34387-34394, 2003.

23. Kim HJ and Kim SH: Tanshinone IIA enhances BMP-2stimulated commitment of $\mathrm{C} 2 \mathrm{C} 12$ cells into osteoblasts via $\mathrm{p} 38$ activation. Amino Acids 39: 1217-1226, 2010.

24. Sohn HS, Heo JS, Kim HS, Choi Y and Kim HO: Duration of in vitro storage affects the key stem cell features of human bone marrow-derived mesenchymal stromal cells for clinical transplantation. Cytotherapy 15: 460-466, 2013.

25. Jang SI, Jeong SI, Kim KJ, Kim HJ, Yu HH, Park R, Kim HM and You YO: Tanshinone IIA from Salvia miltiorrhiza inhibits inducible nitric oxide synthase expression and production of TNF-alpha, IL-1beta and IL-6 in activated RAW 264.7 cells. Planta Med 69: 1057-1059, 2003.

26. Lee SY, Choi DY and Woo ER: Inhibition of osteoclast differentiation by tanshinones from the root of Salvia miltiorrhiza Bunge. Arch Pharm Res 28: 909-913, 2005.

27. Wang X, Wei Y, Yuan S, Liu G, Lu Y, Zhang J and Wang W: Potential anticancer activity of tanshinone IIA against human breast cancer. Int J Cancer 116: 799-807, 2005.

28. Ménard $C$ and Tarte K: Immunoregulatory properties of clinical grade mesenchymal stromal cells: Evidence, uncertainties, and clinical application. Stem Cell Res Ther 4: 64, 2013.

29. Simeone A, Acampora D, Pannese M, D'Esposito M, Stornaiuolo A, Gulisano M, Mallamaci A, Kastury K, Druck T and Huebner K: Cloning and characterization of two members of the vertebrate Dlx gene family. Proc Natl Acad Sci USA 91: 2250-2254, 1994.

30. Erceg I, Tadić T, Kronenberg MS, Marijanović I and Lichtler AC: Dlx 5 regulation of mouse osteoblast differentiation mediated by avian retrovirus vector. Croat Med J 44: 407-411, 2003.

31. Lee MH, Kim YJ, Yoon WJ, Kim JI, Kim BG, Hwang YS, Wozney JM, Chi XZ, Bae SC, Choi KY, et al: Dlx5 specifically regulates Runx 2 type II expression by binding to homeodomainresponse elements in the Runx2 distal promoter. J Biol Chem 280: 35579-35587, 2005.

32. Zhang Z, Song Y,Zhang X, Tang J, Chen J and Chen Y: Msx1/Bmp4 genetic pathway regulates mammalian alveolar bone formation via induction of Dlx5 and Cbfa1. Mech Dev 120: 1469-1479, 2003. 